\title{
Effects of recombinant bovine interleukin-8 (rblL-8) treatment on health, metabolism, and lactation performance in Holstein cattle I: Production and functional characterization of rblL-8 in vitro and in vivo
}

\author{
M. L. S. Bicalho, M. Zinicola, V. S. Machado, F. S. Lima, A. G. V. Teixeira, C. Narbus, M. R. Xavier, \\ H. Higgins, and R. C. Bicalho* \\ Department of Population Medicine and Diagnostic Sciences, Cornell University, Ithaca, NY 14853-6401
}

\section{ABSTRACT}

In the present study, we standardized processes of cloning and purification of recombinant bovine interleukin-8 (rbIL-8) from bacterial culture and assessed its biological activity in Holstein cattle. Plasmid containing a subclone of bovine IL-8 was expressed using Escherichia coli BL21 and cell lysate was purified by chromatography. The presence of rbIL- 8 was assessed by Western blot analyses and function was confirmed in vitro using a chemotaxis chamber. Based on optical density values, chemoattractant properties of rbIL-8 were 10-fold greater compared with control wells. Two in vivo studies were conducted to assess the biological activity of rbIL8. For study 1, one-year-old Holstein heifers $(n=20)$ were randomly allocated to receive a single intravaginal administration containing $1,125 \mu \mathrm{g}$ of rbIL-8 diluted in $20 \mathrm{~mL}$ of saline solution (rbIL-8, $\mathrm{n}=10)$ or a single intravaginal administration of 20 $\mathrm{mL}$ of saline solution (control, $\mathrm{n}=10$ ). For study 2, nonpregnant lactating Holstein cows $(\mathrm{n}=31)$ were randomly allocated to receive an intrauterine administration with $1,125 \mu \mathrm{g}$ of rbIL- 8 diluted in $20 \mathrm{~mL}$ of saline solution (rbIL-8, $\mathrm{n}=11$ ), a positive control consisting of resin-purified lysate of $E$. coli BL21 not transfected with the plasmid coding for rbIL-8 diluted in $20 \mathrm{~mL}$ of saline solution $(E$. coli, $\mathrm{n}=10)$, and a negative control administered with $20 \mathrm{~mL}$ of saline solution (control, $\mathrm{n}$ $=10$ ). An increase in vaginal neutrophils was observed in heifers treated with rbIL- 8 within $3 \mathrm{~h}$ of treatment, but not in control heifers. Additionally, intrauterine administration of rbIL-8 increased the proportion of PMN cells in uterine cytological samples from $3.5 \%$ before treatment to $75.8 \% 24 \mathrm{~h}$ later - an increase that was not observed in the negative control group and cows treated with resin-purified lysate of $E$. coli. To further

Received January 19, 2019.

Accepted May 14, 2019.

*Corresponding author: rcb28@cornell.edu evaluate the effect of local and systemic rbIL- 8 stimulation on the dynamics of circulating white blood cells, a third study was conducted. In study 3 , nonpregnant 8-mo-old Holstein heifers $(\mathrm{n}=30)$ were randomly allocated into 1 of 3 treatment groups: intravenous rbIL- 8 $(1,125 \mu \mathrm{g}$ of rbIL-8 diluted in $5 \mathrm{~mL}$ of saline solution, $\mathrm{n}=10)$; intravaginal rbIL-8 $(1,125 \mu \mathrm{g}$ of rbIL-8 diluted in $20 \mathrm{~mL}$ of saline solution; $\mathrm{n}=10$ ); or intravaginal saline (20 $\mathrm{mL}$ of saline solution, $\mathrm{n}=10)$. Intravenous injection of rbIL- 8 resulted in a transient increase in rectal temperature, which was greater at $2 \mathrm{~h}$ after treatment compared with cows treated intravaginally with rbIL-8 or heifers treated with saline solution. Heifers treated with rbIL-8 intravenously displayed a marked reduction in neutrophils, basophils, lymphocytes, and monocytes within the first $4 \mathrm{~h}$ posttreatment compared with heifers treated intravaginally. However, at $6 \mathrm{~h}$ after treatment, heifers treated with rbIL-8 intravenously displayed a rebound in white blood cell counts caused by an increase in neutrophil counts. These results show that the presented purification method is effective and results in biologically active rbIL- 8 that can be used safely to modulate immune responses in cattle.

Key words: interleukin-8, neutrophil, Holstein cattle

\section{INTRODUCTION}

Interleukin- 8 belongs to the family of chemokines and is secreted by monocytes, activated neutrophils, endothelial cells, and epithelial cells (Baggiolini et al., 1989; Caswell et al., 1999). The binding of IL-8 to its receptors (CXCR1 and CXCR2) on the neutrophil's surface induces activation, simulates chemotaxis, and increases phagocytosis and killing ability (Mitchell et al., 2003). Interleukin-8 is also known to be secreted by the human uterus and placenta, with maximal secretion at the end of gestation (Laham et al., 1999; Elliott et al., 2000). The nucleotide sequence of the bovine IL- 8 cDNA was determined by Morsey et al. (1996) 2 decades ago and was successfully expressed 
in Escherichia coli as a $\beta$-galactosidase fusion protein. Functional characterization of the protein was reported by Caswell et al. (1999), who cloned the same cDNA described by Morsey et al. (1996) into a different expression vector (pGEX-2T).

The functionality of recombinant bovine interleukin-8 (rbIL-8) was also evaluated by Mitchell et al. (2003) who showed that instillation of rbIL- 8 into the bovine lung using a bronchoscope resulted in strong neutrophil recruitment and infiltration. Histologically, neutrophils filled the alveoli and bronchioles, and scattered macrophages contained neutrophils exhibiting morphological features of apoptosis. Prolonged in vitro or in vivo exposure to rbIL- 8 enhanced the subsequent oxidative burst and phagocytic responses of bovine neutrophils (Mitchell et al., 2003). Early influx of neutrophils and enhanced neutrophil activity are associated with reduced incidence of uterine disorders and are essential for clearance of mastitis pathogens (Paape et al., 2002).

Postpartum diseases in dairy cows, especially metritis and mastitis, are highly prevalent in dairy cows worldwide, causing economic losses due to reduction in production outcomes and to increased use of antibiotics and other treatment costs. These inflammatory conditions are also serious animal welfare concerns, resulting in behavioral changes and reduced pain sensitivity thresholds (Sheldon et al., 2006; Peters et al., 2015; Stojkov et al., 2015). Several studies have reported reduced neutrophil function around the time of calving, including decreased chemotaxis, phagocytosis, and killing ability in high-producing dairy cows (Kehrli et al., 1989; Goff and Horst, 1997; Hammon et al., 2006), particularly those that developed uterine diseases. In addition, reduced concentrations of leukocyte chemoattractant were documented by Heuwieser and Grunert (1987) in placentomes sampled at calving and $3 \mathrm{~h}$ after calving in cows with retained fetal membranes (RFM). In fact, the pioneering work of Gunnink established a relationship between RFM and reduced chemotactic activity of leukocytes (Gunnink, 1984a,b). More recently, Kimura et al. (2002) demonstrated that the neutrophils of cows affected with RFM had reduced chemotactic activity and that plasma concentrations of IL-8 throughout the periparturient period were smaller in RFM cows than in non-RFM herdmates beginning at $10 \mathrm{~d}$ before parturition. Furthermore, cows with elevated neutrophil counts in the uterus during the early postpartum period have reduced risks of bacterial infection (Gilbert et al., 2005).

Given the importance of the coordinated neutrophil response to inflammatory conditions, and the role of IL-8 in recruiting neutrophils to the site of infection and stimulating its activity, we aimed to produce a functional rbIL-8 molecule that promotes neutrophil recruitment without exerting detrimental effects on animal health and welfare parameters. Therefore, the objectives of this study were to validate processes to clone, express, and purify rbIL- 8 to assess its biological activity and safety.

\section{MATERIALS AND METHODS}

\section{Ethics Statement}

This study was carried out in strict accordance with the recommendations of The Animal Welfare Act of 1985 (public law 99-198; https://www.nal.usda.gov/ awic/public-law-99-198-food-security-act-1985-subtitle -f-animal-welfare). The research protocol was reviewed and approved by the Institutional Animal Care and Use Committee of Cornell University (protocol number 2013-0039).

\section{Cloning, Production, and Purification of rblL-8}

Plasmid Construction. Construct pET28-HisL-EK-IL8 was created by subcloning the bovine IL-8 cDNA from Trc-His-L-EK-IL8 into pET28A (Novagen, Darmstadt, Germany) using the restriction sites NheI and XhoI. The original Trc plasmid was constructed by PCR amplification of the codon-optimized bovine IL-8 cDNA $\triangle \mathrm{SS}$ using the following primers: 5'-CGGCGCCGTGCTGTCTCGTATGTCCACCGAAC and 5'-GCTCGAGTCACGGATCTTGTTTTTCTGCACG. The PCR product was TA-cloned into pGEM $\mathrm{T}$ vector (Promega, Madison, WI), isolated by blue and white screening, and subsequently sequenced for confirmation. The correct clone was then digested with the restriction enzymes $S f o \mathrm{I}$ and $\mathrm{XhoI}$ and ligated into pTrcHis B vector (Invitrogen, Carlsbad, CA). To maintain a native version of IL-8 upon enterokinase cleavage, the Trc vector was prepared by digestion with $B a m \mathrm{HI}$ followed by digestion with mung bean nuclease to remove the $5^{\prime}$ overhang and create a blunt end for ligation; the vector was then digested with XhoI. The final construct was confirmed by DNA sequencing and cloned into the expression vector E. coli BL21 (Invitrogen).

Fermentation of $E$. coli BL21 Containing the pET28-His-L-EK-IL8 Plasmid. Fermentation was performed using a Bioflo and Celligen 310 Fermentor/ Bioreactor unit (New Brunswick Scientific, Edison, NJ) using a fed-batch fermentation method. Bioengineered E. coli BL21, containing the pET28-His-L-EK-IL8 plasmid, was cultured in chemically defined medium according with $\mathrm{Li}$ and Sha (2015). The composition of the initial fermentation medium was $450 \mathrm{~mL}$ of $10 \times$ phosphate/citric acid buffer $\left[133 \mathrm{~g} / \mathrm{L}\right.$ of $\mathrm{KH}_{2} \mathrm{PO}_{4}, 40 \mathrm{~g} / \mathrm{L}$ of 
$\left(\mathrm{NH}_{4}\right)_{2} \mathrm{HPO}_{4}$, and $17 \mathrm{~g} / \mathrm{L}$ of citric acid] and $4.05 \mathrm{~L}$ of deionized (DI) water. The vessel containing the initial medium was autoclaved for $40 \mathrm{~min}$. After the vessel was removed from the autoclave, the medium was cooled to room temperature and the following ingredients were added: $45 \mathrm{~mL}$ of $240 \mathrm{~g} / \mathrm{L}$ of $\mathrm{MgSO}_{4}, 1.02 \mathrm{~mL}$ of $20 \mathrm{~g} / \mathrm{L}$ of thiamine, $45 \mathrm{~mL}$ of $100 \times$ trace element solution, and $66 \mathrm{~mL}$ of $70 \%$ glucose solution. The concentrated trace element solution was prepared, filter sterilized, and contained the following: $10 \mathrm{~g} / \mathrm{L}$ of iron (III) citrate, $0.25 \mathrm{~g} / \mathrm{L}$ of $\mathrm{CoCl}_{2} \cdot 6 \mathrm{H}_{2} \mathrm{O}, 1.5 \mathrm{~g} / \mathrm{L}$ of $\mathrm{MnCl}_{2} \cdot 4 \mathrm{H}_{2} \mathrm{O}$, $0.15 \mathrm{~g} / \mathrm{L}$ of $\mathrm{CuCl}_{2} \cdot 6 \mathrm{H}_{2} \mathrm{O}, 0.3 \mathrm{~g} / \mathrm{L}$ of $\mathrm{H}_{3} \mathrm{BO}_{3}, 0.25 \mathrm{~g} / \mathrm{L}$ of $\mathrm{Na}_{2} \mathrm{MoO}_{4} \cdot 2 \mathrm{H}_{2} \mathrm{O}, 1.3 \mathrm{~g} / \mathrm{L}$ of zinc acetate $2 \mathrm{H}_{2} \mathrm{O}$, and $0.84 \mathrm{~g} / \mathrm{L}$ of EDTA. Finally, kanamycin was added to the initial medium to a final concentration of $30 \mu \mathrm{g} /$ $\mathrm{mL}$. A concentrated feeding medium was prepared in a 2-L glass bottle; $197.1 \mathrm{~mL}$ of $240 \mathrm{~g} / \mathrm{L} \mathrm{MgSO}_{4}, 7.47 \mathrm{~mL}$ of $20 \mathrm{~g} / \mathrm{L}$ thiamine solution, $67.5 \mathrm{~mL}$ of $100 \times$ trace element solution, and $70 \%$ glucose solution were added to a final volume of $2 \mathrm{~L}$.

The fermentor system included calibrated probes for $\mathrm{pH}$, temperature, and dissolved oxygen (DO). A solution of $30 \%$ ammonium hydroxide was automatically pumped (pump 1) into the vessel to maintain the $\mathrm{pH}$ at 6.8. A $20 \%$ antifoam solution (Antifoam B Emulsion, aqueous-silicone emulsion, Sigma-Aldrich, St. Louis, MO) was automatically pumped (pump 2) into the vessel as needed. Pump 3 was assigned to the feeding medium. The feeding strategy used maximized the bacterial growth without accumulation of glucose in the medium and maintaining DO levels around 25\%. To maintain DO levels around $25 \%$, a calibrated DO probe was used, and medical-grade oxygen sparging and agitation speed (100-1,200 rpm) were varied as needed.

Escherichia coli BL21 was grown overnight in LuriaBertani agar with kanamycin $(30 \mu \mathrm{g} / \mathrm{mL})$ at $37^{\circ} \mathrm{C}$. A single colony was inoculated into $500 \mathrm{~mL}$ of Luria-Bertani broth (kanamycin $30 \mu \mathrm{g} / \mathrm{mL}$ ) and grown overnight using benchtop shaker incubator at $\left(200 \mathrm{rpm}\right.$ at $\left.37^{\circ} \mathrm{C}\right)$. The fermentor vessel was inoculated with $500 \mathrm{~mL}$ of inoculum (approximately 10\% of the initial fermentation medium volume).

The batch fermentation period lasted for a total of $12 \mathrm{~h}$ (overnight $30^{\circ} \mathrm{C}$ ). Feeding was initiated after overnight cultivation. Pump 3 was manually adjusted during the course of the fermentation to maximize feeding rate while maintaining a 25\% DO and no glucose accumulation. Accumulation of glucose was evaluated hourly throughout the feeding period using DO spikes to ensure that glucose was limited. Samples were taken periodically to monitor the cell growth by measuring the wet cell weight (WCW). For each sample, $30 \mathrm{~mL}$ of culture material was added into a
$50-\mathrm{mL}$ plastic tube and pelleted at $4,200 \mathrm{rpm} / 4^{\circ} \mathrm{C}$ for $40 \mathrm{~min}$. The supernatant was carefully removed, and the WCW was calculated. When the WCW reached approximately $40 \%$, protein expression was induced by adding isopropyl $\beta$-D-1-thiogalactopyranoside at a 1 $\mathrm{m} M$ final concentration. Fermentation temperature was reduced to $25^{\circ} \mathrm{C}$ and the feeding medium supplementation was decreased to $27 \mathrm{~mL} / \mathrm{h}$; the induction phase was completed in $24 \mathrm{~h}$. Induced cells were harvested by centrifugation using a continuous flow centrifuge set at 12,000 $\times g$ (Carr Powerfuge Pilot, Barry-Wehmiller, St. Louis, MO). Cell pellets were resuspended in $1 \times$ buffer (50 $\mathrm{mM} \mathrm{NaH}_{2} \mathrm{PO}_{4} ; 300 \mathrm{mM} \mathrm{NaCl}, \mathrm{pH}$ 7.4). Cells were then sonicated on ice with 5 -min pulses at 5 -min intervals 5 times using a Misonix Ultrasonic Liquid Processor Q500 (QSonica LLC, Newtown, CT) at 80\% amplitude. Sonicated cells were centrifuged $(12,000 \times$ $\mathrm{g} / 30 \mathrm{~min}, 4^{\circ} \mathrm{C}$ ) and the supernatant was stored at $4^{\circ} \mathrm{C}$ for purification.

Samples were collected prefeeding, $12 \mathrm{~h}$ postfeeding, $12 \mathrm{~h}$ postinduction, and $24 \mathrm{~h}$ postinduction to perform SDS-PAGE and Western blot to identify the presence of the target protein.

Protein Purification. The soluble fraction of the supernatant was used for purification of rbIL-8 using a custom packed $60-\mathrm{mL}$ column of Talon Metal Affinity Resin (Clontech Laboratories Inc., Mountain View, CA). Automated multi-dimensional purifications of the His-tagged rbIL-8 were performed using the ÄKTA-pure instrument (GE Healthcare Bio-Sciences, Pittsburgh, PA) controlled by Unicorn 7 software and consisted of 2 steps: immobilized metal-affinity chromatography followed by desalting, as described previously (Sigrell et al., 2003). The clear cell lysate was loaded onto the column $(60 \mathrm{~mL} / \mathrm{min})$ previously equilibrated in buffer A (250 mM sodium phosphate monobasic and $1,500 \mathrm{~m} M \mathrm{NaCl} ; \mathrm{pH}$ 7.4). Protein was eluted using a linear gradient of buffer B (buffer A plus $150 \mathrm{~m} M$ imidazole; pH 7.4); the concentration of buffer B increased from 0 to $100 \%$ over 20 column volumes. Elution liquid was fractionated into $50-\mathrm{mL}$ tubes and fractions were analyzed by SDS-PAGE. Fractions containing the protein were pooled and loaded onto a 1,200-mL Sephadex G-25 (GE Healthcare Bio-Sciences) column for buffer exchange. Sizes exclusion chromatography was performed using Sephacryl S-300 HR SEC resin in HiPrep column (GE Healthcare Bio-Sciences) to estimate the purity of the final rbIL-8. The final protein sample was filter sterilized and stored in $25 \%$ glycerol at $-20^{\circ} \mathrm{C}$. Purified protein was quantified using Quick Start Bradford Protein Assay (Bio-Rad, Hercules, CA), and final concentration was expressed in milligrams per milliliter. 


\section{Western Blot and SDS-PAGE}

Western blot analysis was conduct to assess the presence of $6 \mathrm{xHis}$ tagged IL8 protein in the culture lysate obtained from different production phases. The whole cells lysates were separated on 15\% SDS-PAGE gel followed by electro transfer to nitrocellulose membrane. The membrane was blocked with $5 \%$ skim milk solution at room temperature for $1 \mathrm{~h}$ and probed overnight at $4^{\circ} \mathrm{C}$ with $6 x H i s$ monoclonal antibody (albumin free, ClonTech, Mountain View, CA) prepared in a dilution of 1:5,000. Subsequently, the membrane was washed with Tris-buffered saline with Tween $20(20 \mathrm{~m} M$ Tris, $150 \mathrm{mM} \mathrm{NaCl}, 0.01 \%$ Tween 20) and treated with goat anti-mouse IgG antibody horseradish peroxidase conjugate (Sigma-Aldrich) diluted 1:2,500 for $1 \mathrm{~h}$ at room temperature, shaking. Blot was developed using 1-Step chloronaphthol substrate solution (Thermo Fisher Scientific, Waltham, MA). The SDS-PAGE gels with whole cells lysates and purified protein were also stained and destained using Coomassie Brilliant Blue R-250 (Bio-Rad) and destaining solution (10\% acetic acid and $45 \%$ methanol).

\section{Determination of Endotoxins}

In this work, the endotoxin content was verified with the ToxinSensor Chromogenic LAL Endotoxin Assay Kit (Genscript, Piscataway, NJ). This test was performed, and the concentrations of endotoxin were calculated according to the manufacturer's guidelines with the formula $0.2618 \times \mathrm{x}-0.0012$, where $\mathrm{x}$ is the absorbance at $545 \mathrm{~nm}$ of each sample (fractions containing the protein were concentrated). In all cases, a total of $80 \mu \mathrm{g}$ of protein was used. The initial 80 $\mu \mathrm{g}$ was then diluted 1:100,1:1,000, and 1:10,000 with endotoxin-free water.

\section{In Vitro Assessment of Neutrophil Chemotaxis Induced by rb/L-8}

Bovine blood PMN cells were isolated as described by Roth and Kaeberle (1981). In brief, blood samples were centrifuged for $20 \mathrm{~min}$ at $1,000 \times \mathrm{g}$ at $4^{\circ} \mathrm{C}$ and the plasma and buffy coat aspirated and discarded. Polymorphonuclear cells were separated from the remaining packed red blood cells by using 2-volume hypotonic lysis. The PMN cells (primarily neutrophils, but also basophils and eosinophils) were adjusted to a final concentration of $6.0 \times 10^{7}$ cells $/ \mathrm{mL}$ in Hanks' Balanced Salt Solution (Grand Island Biological Co., Grand Island, NY) at room temperature until they were subjected to functional assays.
The chemotactic assay was conducted in a 96-well chemotaxis chamber (Neuro Probe MBA96, Neuro Probe Inc., Gaithersburg, MD) fitted with a $5-\mu \mathrm{m}$ framed filter following the manufacturer's instructions and as described previously (Shi et al., 1993). The framed filter is a polycarbonate membrane bonded to a rigid flame possessing a pressure-sensitive adhesive on the lower side. The 96-well chemotaxis chamber has a lower plate with a recess that accepts a microtiter plate, an upper plate with 96 through-holes, and a silicone rubber gasket secured to the underside of the upper plate with retention pins. The upper and the lower plates are attached to each other with a hinge on one side and latches on the opposite side. To set up the apparatus, a microtiter plate was filled $(400 \mu \mathrm{L})$ with medium containing chemoattractant or medium alone and placed in the recess of the lower plate. The framed filter was then placed on top of the filled microtiter plate with the adhesive side down. The upper plate, with the gasket installed, was then closed on the filter and latched to the lower plate. Then $200 \mu \mathrm{L}$ of cell suspension (between $6 \times 10^{4}$ and $6 \times 10^{6}$ cells) in PBS was added to the wells of the upper plate. Assays were carried out at $37^{\circ} \mathrm{C}$ in $5 \% \mathrm{CO}_{2} / 95 \%$ air for $2 \mathrm{~h}$. Upon completion of incubation, the medium in the wells of the upper plate was replaced with $200 \mu \mathrm{L}$ of PBS-EDTA and incubated at $4^{\circ} \mathrm{C}$ for $30 \mathrm{~min}$. After incubation, excess fluid and leftover cells were removed with cotton swabs. The microplate containing the filter was centrifuged at $500 \times g$ at 18 to $21^{\circ} \mathrm{C}$ for $10 \mathrm{~min}$ to pellet cells that had migrated into or through the filter into the lower compartment wells in response to rbIL-8.

Quantification of cells that responded to chemotaxis was performed using a rapid colorimetric assay based on reduction of 3-(4,5-dimethylthiazol-2-yl)-2,5-diphenyltetrazolium bromide (MTT; Sigma-Aldrich), which measures living cells (Mosmann, 1983). Reduced MTT was measured based on optical density at $540 \mathrm{~nm}$ using a microplate reader (BioTek Instruments, Winooski, VT).

\section{In Vivo Assessment of Neutrophil Chemotaxis Induced by rb/L-8}

Three experiments were conducted to assess the in vivo biological activity of rbIL- 8 in treating animals based on recruitment of PMN into the reproductive tract, and to assess the effect of local and systemic rbIL-8 stimulation on the dynamics of circulating white blood cells. Given the possibility that the effects of rbIL- 8 are dependent on animal's age and physiological status, in vivo chemotaxis trials were conducted using 1-yr-old Holstein heifers and nonpregnant lactating Holstein cows. 
Animals and Facilities. Holstein heifers and lactating cows from a single farm located near Ithaca, New York, were used in this study within the period of March to June 2014. The farm milked 3,300 Holstein cows 3 times daily in a double 52-stall parallel milking parlor. Cows were housed in freestall barns equipped with sprinklers, fans, and sand-bedded stalls. Animals had ad libitum access to water and received a TMR formulated to meet or exceed nutritional requirements of each category.

Study Design and Data Collection. In study 1, 1-yr-old Holstein heifers $(\mathrm{n}=20)$ were randomly allocated to receive a single intravaginal administration containing $1,125 \mu \mathrm{g}$ of rbIL- 8 diluted in $20 \mathrm{~mL}$ of saline solution (rbIL-8; $\mathrm{n}=10$ ) or $20 \mathrm{~mL}$ of sterile saline solution (control; $\mathrm{n}=10$ ) on study d 0 . Initial dose of rbIL-8 was based on data from other species in which a dose of $25 \mu \mathrm{g} / \mathrm{kg}$ has previously been shown to be effective in rabbits (Ley et al., 1993), and suggested to elicit significant local or systemic inflammatory reactions, or both (Watanabe et al., 2012). Animal health status was monitored continuously by one of the veterinarians of the research team for the first $12 \mathrm{~h}$ after treatment and once daily until study d 4 . Animals were evaluated for rectal temperature (fever $>39.5^{\circ} \mathrm{C}$ ), respiratory pattern $(0=$ breathing slowly and evenly; $1=$ panting or breathing with obvious labor; $2=$ rapid breathing), attitude $(0=$ alert; $1=$ depressed; $2=$ nonresponsive $)$, hydration status $(0=$ hydrated; $1=$ skin tented 2 to $5 \mathrm{~s} ; 2=$ skin tented 6 to $10 \mathrm{~s} ;$ and $3=$ skin tented $\geq 10 \mathrm{~s}$ ), and signs of pain or discomfort. Potential behavioral signs of pain were recognized and evaluated according to Forkman pain chart evaluation (Gleerup et al., 2015). Treatment was performed after cows were restrained in headlock stanchions, the external genital area of cows was washed with warm water and soap and dried with paper towels followed by a $30 \%$ iodine spray before treatment. The rbIL-8 or sterile saline solution was gently administered into the vaginal tract with individually wrapped sterile infusion tubes (Continental Plastic Corp., Delavan, WI) capped with a 5-mL screw- tip sterile syringe (Becton, Dickinson and Company, Franklin Lakes, NJ) and deposited at the cranial aspect of the vagina. Cytological evaluation of the vaginal epithelium was performed before administration and $3 \mathrm{~h}$ later using a cytobrush (Cytobrush Plus GT, CooperSurgical, Trumbull, CT) attached to a stainless-steel gun adapted for use in cattle (Kasimanickam et al., 2004). In brief, study cows were restrained in a head-lock stanchion, and the perineum and vulva were cleansed with a paper towel and disinfected with $70 \%$ ethanol before sample collection. The lips of the vulva were opened and the cytobrush gun was inserted into the vagina. After swabbing (by gently rubbing or rolling) the vaginal wall, the applicator is removed and rolled (not smeared) onto a glass slide. Routine DiffQuik (Dade Diagnostics, West Monroe, LA) staining was performed after air-drying the slide. Each slide was examined by the same examiner under bright-field microscopy at $400 \times$ magnification to aid in particular cell identification. The examiner counted 200 cells from each slide and the percentage of PMN among all other cells was determined.

In study 2, to ensure that the biological effects observed after treatment were in response to rbIL- 8 and not to other proteins expressed by the vector, nonpregnant lactating Holstein cows $(\mathrm{n}=31)$ were allocated randomly to receive an intrauterine administration with $1,125 \mu \mathrm{g}$ of rbIL- 8 diluted in $20 \mathrm{~mL}$ of saline solution (rbIL-8; n = 11), resin-purified lysate of E. coli BL21 that was not transfected with the plasmid coding for rbIL-8 diluted in $20 \mathrm{~mL}$ of saline solution $(E$. coli; $\mathrm{n}=$ 10), and a negative control administered with $20 \mathrm{~mL}$ of saline solution (control, $\mathrm{n}=10$ ). Intrauterine administration of purified lysate of $E$. coli BL21 (free of the plasmid coding for rbIL-8) was prepared as using the same technique that was described for the rbIL-8 preparation. Cows were restrained in headlock stanchions and the perineal area and vulva were cleansed with paper towels and disinfected with $70 \%$ ethanol before treatments were applied. A sterile uterine administration pipette was manipulated into the uterus, the tip was exposed to the uterine lumen, and the treatment was administered into the uterus. Cytological evaluation of the endometrium was performed before administration and $24 \mathrm{~h}$ after using a cytobrush as described in study 1. In brief, the lips of the vulva were opened and the cytobrush gun was inserted into the vagina and passed through the cervix. Once inside of the uterine body, the cytobrush was exposed, rotated clockwise a full 360 degrees to obtain cellular material from the endometrium, and then withdrawn inside of the gun. Slides for cytological examination were prepared on farm by rolling the cytobrush on a clean glass microscope slide and air-dried before being stained with modified Wright-Giemsa stain (Diff-Quik 1, Dade Diagnostics). Slides were examined as described in study 1 .

Study 3 consisted of a single dose of rbIL- 8 via 2 different routes (intravenous and intravaginal) to evaluate the effect of local and systemic rbIL- 8 stimulation on the dynamics of circulating white blood cells. In total, 30 nonpregnant 8-mo-old Holstein heifers were randomly allocated into 1 of 3 treatments: intravenous rbIL-8 $(\mathrm{n}=10)$, intravaginal rbIL-8 $(\mathrm{n}=10)$, or intravaginal saline $(\mathrm{n}=10)$. The intravenous dose was composed of $1,125 \mu \mathrm{g}$ of rbIL- 8 diluted in $5 \mathrm{~mL}$ of saline solution administered into a jugular vein. The intravaginal treatment contained the same dose of $1,125 \mu \mathrm{g}$ of rbIL- 
8 diluted in $20 \mathrm{~mL}$ of saline solution or $20 \mathrm{~mL}$ of saline solution. Intravaginal treatments were administered as described in study 1.

Animal health status (i.e., respiratory pattern, attitude, hydration status, and signs of pain or discomfort) was monitored continuously by one of the veterinarians of the research team for the next $12 \mathrm{~h}$ after treatment and once daily until the last day of the study at d 14 . Rectal temperature was recorded right before treatment $(0 \mathrm{~h})$ and at 2, 6, and $24 \mathrm{~h}$. For determination of differential leukocyte counts, blood samples were collected at enrollment and at $10 \mathrm{~min}, 2 \mathrm{~h}, 4 \mathrm{~h}, 6 \mathrm{~h}, 1 \mathrm{~d}, 2 \mathrm{~d}, 7 \mathrm{~d}$, and $14 \mathrm{~d}$ posttreatment from the coccygeal vein/artery were drawn into 2-mL syringe and transferred immediately into commercial 2-mL Vacutainer tubes containing lithium-heparin (Becton Dickinson and Company, Franklin Lakes, NJ). Blood samples were submitted to the New York State Veterinary Diagnostic Laboratory for complete blood count analysis.

\section{Statistical Analyses}

Continuous variables were analyzed by ANOVA using the GLIMMIX procedure of SAS version 9.3 (SAS Institute Inc., Cary, NC). Results from in vitro chemotactic assays were analyzed by one-way ANOVA. Data from in vivo studies were analyzed by repeated measures ANOVA. Treatment, time, and the interaction of treatment and time were included as fixed effects into the statistical models, whereas animal identification number was added as a random effect. For studies with more than 2 treatments, pairwise comparisons were performed using Tukey's method. Tests for normality of residuals and homogeneity of variances were assessed using residual plots. Results are presented as LSM and SEM. Differences with $P \leq 0.05$ were considered significant and those with $0.05<P \leq 0.10$ were considered tendencies.

\section{RESULTS}

\section{Expression and Purification of Recombinant Bovine IL-8}

Herein, an E. coli BL21 expressing rbIL-8 gene under the control of T-7 promoter was developed. The clone was cultivated in the bioreactor for production of rbIL8 . The clone was initially batch fermented for a period of $12 \mathrm{~h}$, followed by a fed-batch period of $12 \mathrm{~h}$. At the end of the fed-batch period, the WCW reached 50\% (500 $\mathrm{g} / \mathrm{L}$; Figure 1A). Expression of the rbIL-8 protein was analyzed by $12 \%$ Tris-glycine SDS-PAGE stained with Coomassie brilliant blue and confirmed by Western blot analyses using a monoclonal antibody against bovine native IL-8 (Thermo Fisher Scientific). The SDS-PAGE and Western blot analysis revealed the presence of the $\sim 12 \mathrm{kDa}$ rbIL- 8 protein. The results showed that rbIL8 was expressed successfully with maximum expression of rbIL- 8 within $12 \mathrm{~h}$ after induction (Figure $1 \mathrm{~B}$ and 1C). Size exclusion HPLC analysis using a prepacked HiPrep 26/60 column (Sephacryl S-300 HR) confirmed a purity of $>97 \%$ of the rbIL-8 (Figure 1D and 1E).

\section{Chemotactic Activity of rblL-8}

In Vitro Chemotactic Assays. Results from in vitro chemotactic assays confirmed the bioactivity of rbIL-8. Based on optical density values, chemoattractant properties of rbIL-8 was 10 -fold greater $(P \leq 0.05)$ compared with control wells (Figure 2A).

In Vivo Chemotactic Assays. In study 1, similar to in vitro results, an increase in vaginal PMN was observed in heifers treated with rbIL- 8 within $3 \mathrm{~h}$ of treatment, but not in control heifers (Figure 2B).

In study 2, intrauterine administration of rbIL-8 increased the proportion of PMN cells in uterine cytological samples from $3.5 \%$ before treatment to $75.8 \%$ $24 \mathrm{~h}$ later, an increase that was not observed in untreated controls and cows treated with resin-purified lysate of $E$. coli BL21 that was not transfected with the plasmid coding for rbIL-8 (Figure 2C). These results indicate that the rbIL-8 is biologically active and successfully induced neutrophil activation, chemotaxis, and diapedesis both in vitro and in vivo.

In the route administration trial in study 3, intravenous injection of rbIL- 8 resulted in a transient increase in rectal temperature, which was only observed at the measurement taken $2 \mathrm{~h}$ after treatment compared with heifers treated with rbIL-8 intravaginally or untreated controls (Figure 3). No difference in rectal temperature among groups was observed at the measurements taken 6 and $24 \mathrm{~h}$ posttreatment. Respiratory pattern, attitude, hydration status, and signs of pain or discomfort were not affected by treatment and remained within normal limits for the duration of the study.

White blood cell counts in control animals and those treated with rbIL-8 intravaginally were stable during the entire experimental period (Figure 4A). On the other hand, animals treated with rbIL-8 intravenously displayed a marked reduction in white blood cells $2 \mathrm{~h}$ after injection that was sustained until $4 \mathrm{~h}$ posttreatment. Such reduction was driven by a decrease in blood counts of neutrophils (Figure 4B), lymphocytes (Figure $4 \mathrm{C}$ ), monocytes (Figure 4D), and basophils (Figure $4 \mathrm{E})$. Basophil counts in heifers treated with rbIL-8 intravenously returned to the same levels as the other 2 groups at $4 \mathrm{~h}$ after treatment. Nevertheless, counts of neutrophils, lymphocytes, and monocytes remained 
A

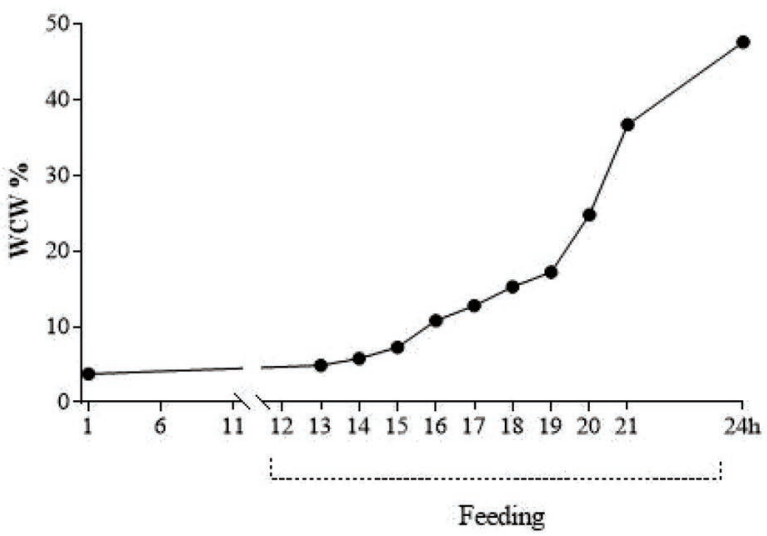

D
B

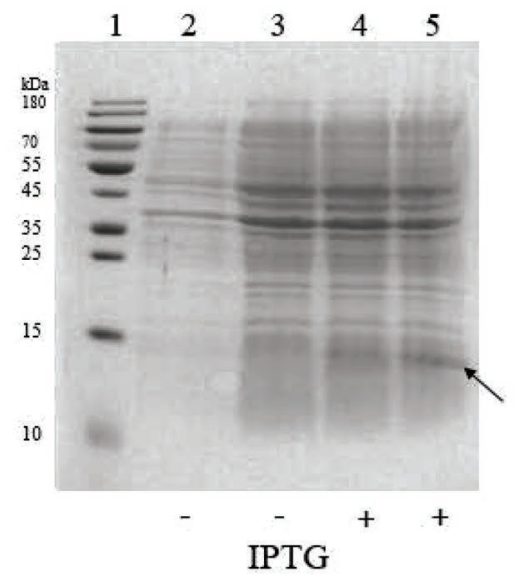

C

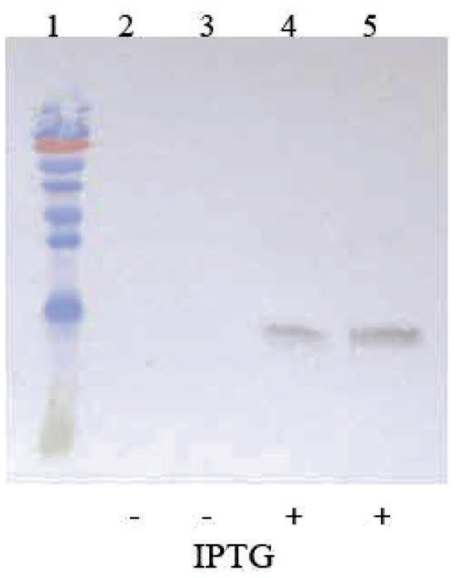

E

12

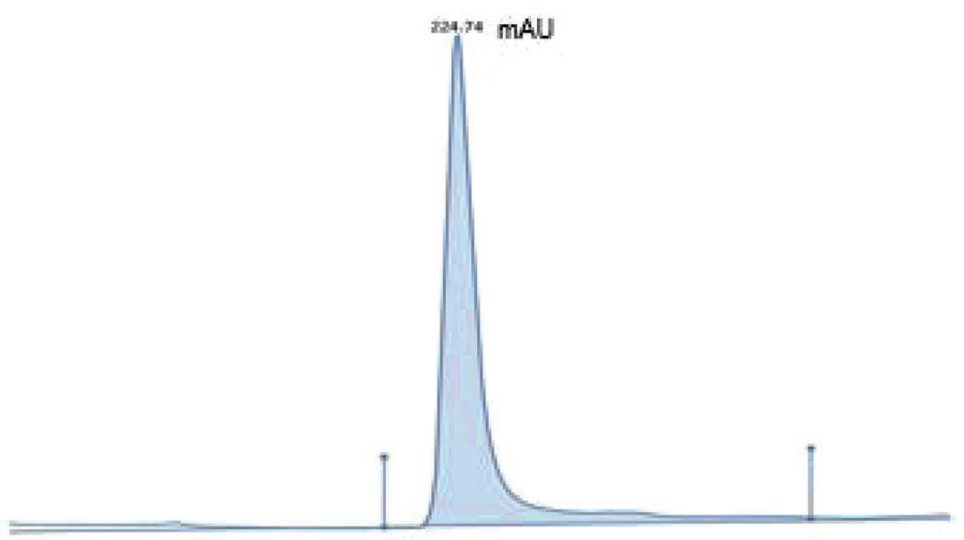

Fractions
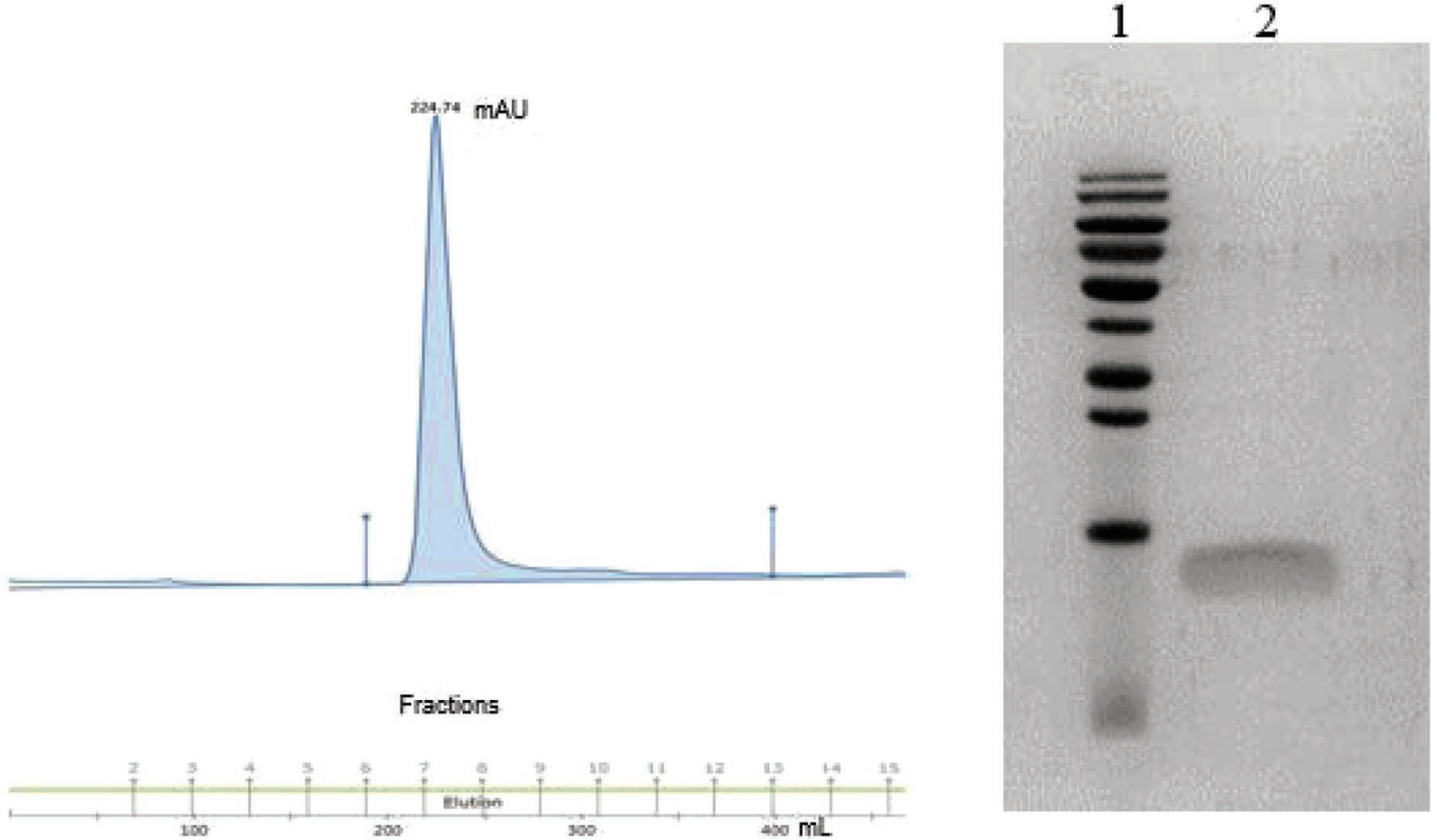

Figure 1. Expression and purification of recombinant bovine interleukin-8 (rbIL-8). (A) Bacteria growth and gain in wet cell weight (WCW\%) by the recombinant BL21-rbIL8 construct cultured in a bioreactor at different time points; (B) SDS-PAGE analysis showing expression of rbIL-8 (arrow); and (C) Western blot probed with antihistidine antibodies showing presence of $6 \mathrm{x}-\mathrm{His}$ tagged rbIL-8 protein, at $12 \mathrm{~h}$ postinoculation (lane 2), $12 \mathrm{~h}$ postfeeding (lane 3), $12 \mathrm{~h}$ post isopropyl $\beta$-D-1-thiogalactopyranoside (IPTG) induction (lane 4 ), and $24 \mathrm{~h}$ post IPTG induction (lane 5); DNA marker at lane 1. (D) Chromatogram showing high concentration and purity of rbIL-8 obtained from size exclusion chromatography process (protein size of $12 \mathrm{kDa}$ ). $\mathrm{mAU}=$ milli-absorbance units. $(\mathrm{E})$ SDS-PAGE analysis confirming the presence of purified rbIL-8: DNA marker (lane 1), recombinant BL21 showing induced band of rbIL-8 (lane 2).

lower $(P<0.05)$ at $4 \mathrm{~h}$ after treatment in heifers treated with rbIL-8 intravenously compared with counterparts treated with rbIL- 8 intravaginally and controls. At 6 $\mathrm{h}$ after treatment, heifers treated with rbIL-8 intravenously displayed a rebound in white blood cell counts (Figure 4A) driven primarily by a dramatic increase in neutrophil counts (Figure 4B).

\section{DISCUSSION}

In the present study, we standardized the process of cloning, production, and purification of rbIL-8 from bacterial culture. We tested different heating temperatures and heating times for partial purification of rbIL-8. We found that partial purification was achieved after heat- 


\section{$\square$ Control $\square$ rblL-8 $\square$ E.coli}
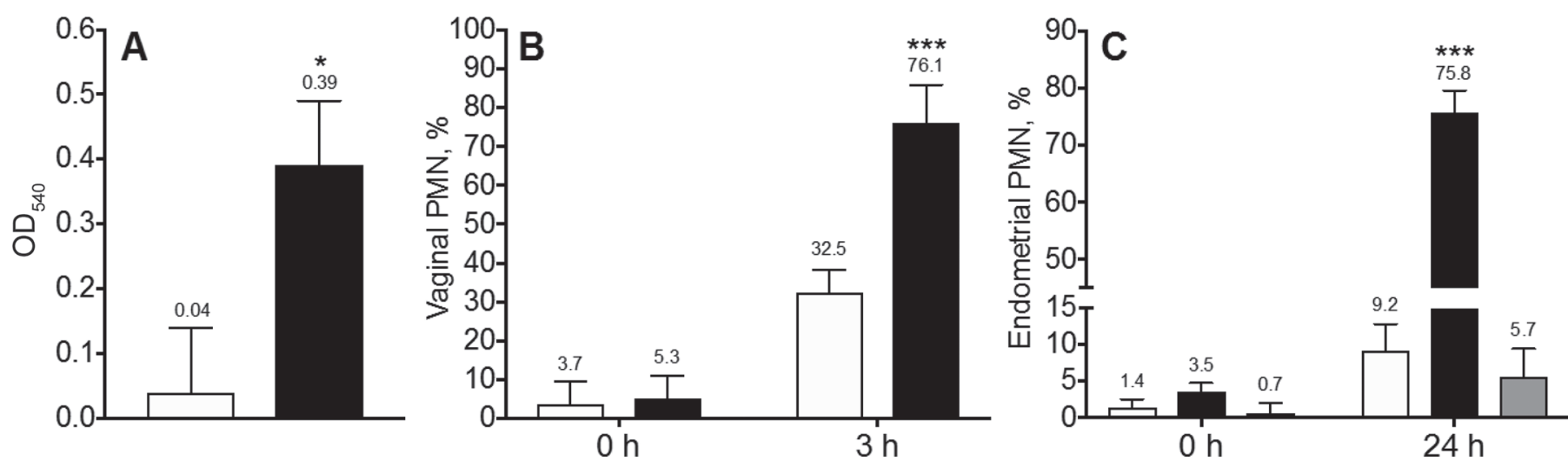

Figure 2. In vitro and in vivo assessment of neutrophil chemotaxis induced by recombinant bovine interleukin-8 (rbIL-8). (A) Effect of rbIL-8 on in vitro chemotaxis was confirmed using a 96-well chemotaxis chamber. For positive controls for chemotaxis, rbIL- 8 was placed into a series of lower compartment wells. Wells with PBS without IL-8 served as negative controls for chemotaxis. Two experiments were conducted to assess in vivo biological activity: (B) 1-yr-old Holstein dairy heifers received a single intravaginal administration containing 1.125 mg of rbIL-8 or sterile saline (control) on the day of parturition, and (C) nonpregnant lactating Holstein cows received an intrauterine administration with $1,125 \mu \mathrm{g}$ of rbIL-8, or resin-purified lysate of Escherichia coli BL21 that was not transfected with the plasmid coding for rbIL-8 or sterile saline (control). Graphs show the proportion of PMN cells detected in vaginal (B) and endometrial (C) cytology samples. Error bars indicate SEM. ${ }^{* * *} P \leq 0.001,{ }^{*} P \leq 0.05$. $\mathrm{OD}_{540}=$ optical density at $540 \mathrm{~nm} .{ }^{* * *} P \leq 0.001,{ }^{* *} P \leq 0.01,{ }^{*} P \leq 0.05$.

ing the bacterial lysate at $70^{\circ} \mathrm{C}$ for 10 min, after which most of the bacterial proteins were removed. Similar results have been reported for human recombinant IL-8 (Hou et al., 2005). After a column chromatography step, this purification procedure resulted in final yields of $1.2 \mathrm{mg}$ of $>95 \%$ pure rbIL- 8 per liter of $E$. coli BL21 culture. Similar results were seen by Caswell et al. (1999), who reported a protein yield of $0.7 \mathrm{mg}$ of purified recombinant bovine IL-8 for every liter of induced E. coli culture. We then conducted functional tests, both in vitro and in vivo, to ensure that rbIL8 was biologically active. The results of our in vitro and in vivo indicated that rbIL- 8 can be successfully produced by $E$. coli BL21 and that the purified protein is biologically active.

Interleukin-8 is a proinflammatory chemokine commonly associated with disease in several species. It is produced by macrophages, smooth muscle cells, epithelial cells, and endothelial cells that recruit neutrophils, basophils, and $\mathrm{T}$ cells to the site of infection (Mitchell et al., 2003). Accordingly, the development of diseaselike signs that impair the well-being and potentially performance was a major concern of treating dairy cows with rbIL-8. We completed 2 studies to evaluate potential negative effects of rbIL-8 treatment in cattle; however, no major adverse effects on health parameters and welfare measures were observed in heifers and lactating cows. Experimental animals that received intrauterine administration of rbIL-8 were monitored constantly in case of signs of disease, but no adverse effects were evident. We also demonstrated that vaginal and intrauterine administration with rbIL- 8 is effective in recruiting neutrophils into the reproductive tract. The efficacy of rbIL-8 as a neutrophil chemoattractant in vivo was previously demonstrated by intradermal skin testing in a normal calf (Baggiolini et al., 1989) and by intratracheal instillation of IL- 8 into the lung (Caswell et al., 1999; Mitchell et al., 2003).

Bacteria-derived factors are particularly suitable for investigation of inflammatory and immune processes because they are often potent immune-stimulators. A study has revealed that several bacterial species secrete substances with chemotactic activity toward leukocytes into the culture supernatant (Tsukamoto et al., 1990). That study showed that bacteriocin purified from a culture supernatant of Streptococcus mutans induced chemotaxis of human PMN and monocytes. Bacterial lipopolysaccharides are among the best understood virulence factors in terms of inducing neutrophil infiltration on examined tissues (Persson Waller, 1997; Johnson et al., 2011; Watanabe et al., 2012). However, in the present study, the presence of such extraneous immune stimulators (i.e., contaminants from rbIL-8 production) able to elicit intrauterine granulocyte recruitment was ruled out. Nonpregnant lactating Holstein cows treated with an intrauterine administration of rbIL-8 underwent a major increase in the proportion of PMN, whereas controls treated with resin-purified lysate of $E$. coli BL21 that did not have the expression plasmid coding for IL-8 did not. Additionally, LPS concentrations in the final purified rbIL- 8 was 8 endotoxin units $/ \mathrm{mL}$, which is considered very low and 


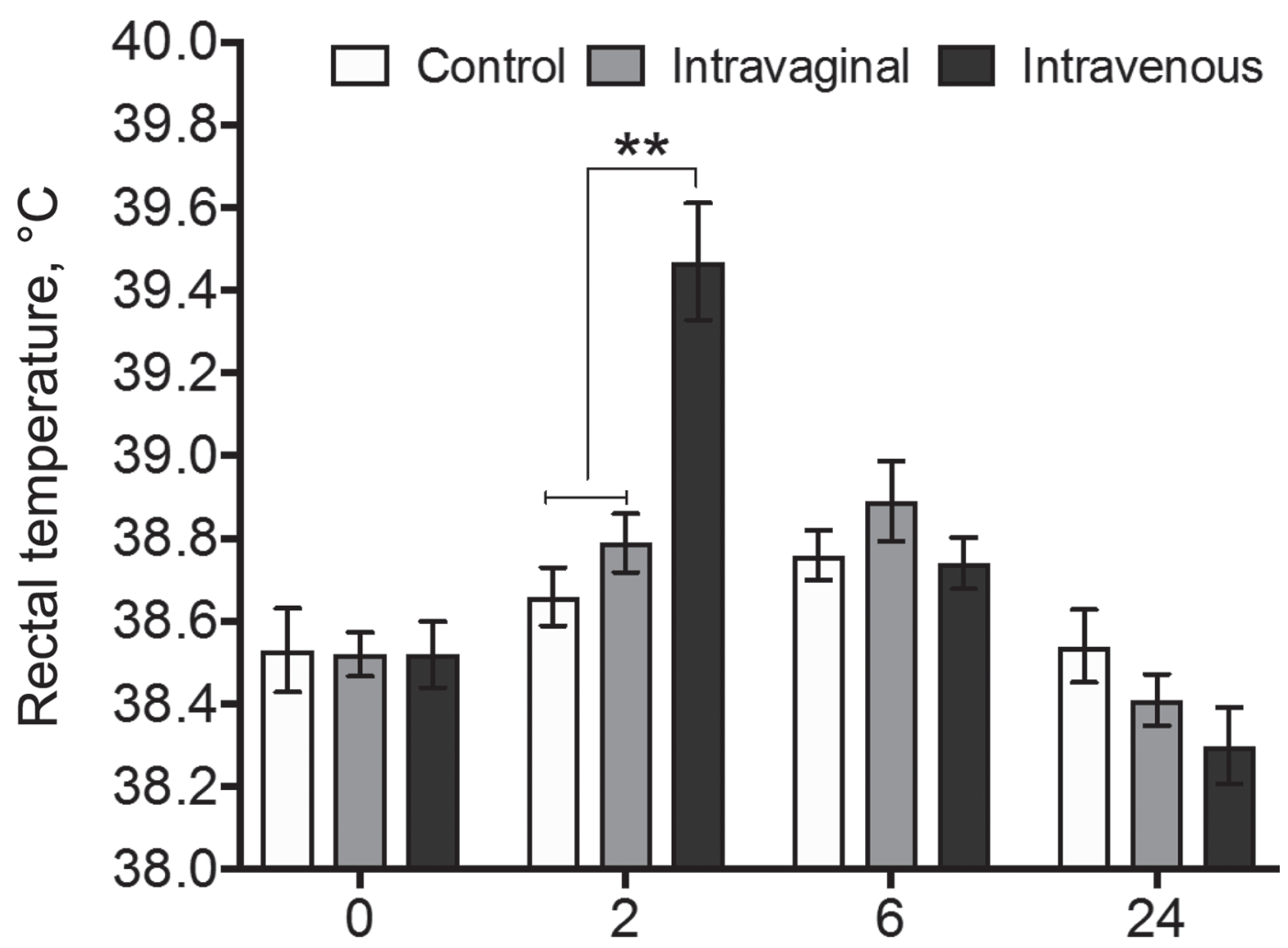

Hours relative to rblL- 8 administration

Figure 3. Rectal temperature $\left({ }^{\circ} \mathrm{C}\right)$ of nonpregnant heifers that received a single intravenous recombinant bovine interleukin- 8 (rbIL-8), intravaginal rbIL- 8 dose, or saline solution at $0 \mathrm{~h}$ (before treatment), $2 \mathrm{~h}, 6 \mathrm{~h}$, and $24 \mathrm{~h}$ relative to the rbIL- 8 administration (study 3 ). Error bars indicate SEM. ${ }^{* *} P \leq 0.01$.

with the recommended range for injectable biologicals (Brito and Singh, 2011). Taken together, these results indicate that the produced rbIL- 8 is highly pure and biologically active and can be used safely to modulate immune responses in cattle.

The elevation in body temperature in response to rbIL-8 observed $2 \mathrm{~h}$ after intravenous treatment with intravenous rbIL- 8 was expected as the pyrogenic effect of IL-8 has been reported previously (Rothwell et al., 1990; Zampronio et al., 1995). Interestingly, in another study by Zampronio et al. (1994) recombinant human IL-8 injected intravenously into rabbits was not pyrogenic, whereas intracerebroventricular injection induced fever, which might be attributed to the lower dosage used in that study. In addition, the range of mediators capable of producing fever in different species, together with their doses and routes of administration, vary widely. For example, intravenous doses of lipopolysaccharide, IL-1 $\beta$, and TNF- $\alpha$ that induce fever in rabbits and humans are smaller than that in rats (Kettelhut and Goldberg, 1988; Zampronio et al., 1994, 2000). Similarly, intravenous administration of IL-6 at doses 20 times higher than that which induces fever in rabbits does not promote fever in rats (Helle et al., 1988; Rothwell et al., 1990).

Intravenous administration of rbIL-8 altered the peripheral blood leukocyte distribution in the present study. Cytokines are well-known promoters of white blood cell differentiation (Zhu and Emerson, 2002). In the present study rbIL- 8 administration decreased the white blood cell population within the first $4 \mathrm{~h}$ after intravenous injection; however, a sustained alteration past this point was not observed. Specifically, the levels of blood neutrophils, basophils, lymphocytes, and monocytes behaved similarly after treatment with a significant drop in white blood cells in the intravenously treated animals observed $2 \mathrm{~h}$ after treatment, followed by a relative increase at $4 \mathrm{~h}$ after treatment. However, neutrophils were the only leukocyte to increase significantly at $6 \mathrm{~h}$ after intravenous rbIL- 8 treatment. The time seen in our study required for the cells after IL-8 treatment, to start migration from the blood vessels into the tissues, may be explained by the positive amplification loop and orchestrated protein synthesis necessary for the neutrophil migration (Manley et al., 2018). On the other hand, the rapidity of count recovery 
at $4 \mathrm{~h}$ postinjection following this significant decrease is indicative of bone marrow reserve granulocytes (Jagels and Hugli, 1992).
In previous studies it was reported that blood was repopulated with granulocytes recruited from the bone marrow and released from the lungs after a single intra-
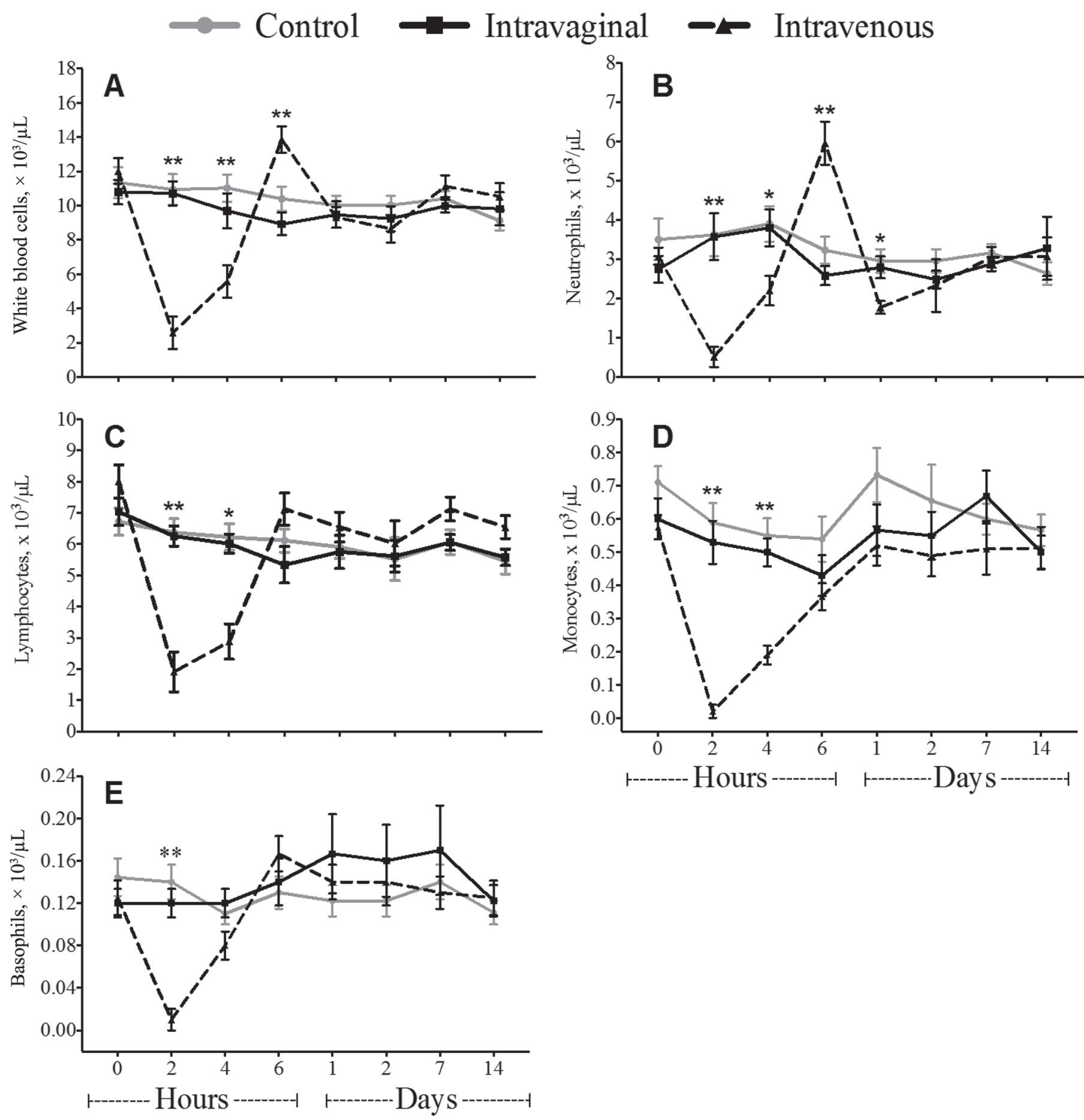

Figure 4. The dynamic of the concentration of white blood cells (A), neutrophils (B), lymphocytes (C), monocytes (D), and basophils (E) of nonpregnant heifers treated with recombinant bovine interleukin-8 (rbIL-8) intravenously or intravaginally, or saline solution (study 3 ). Blood samples were collected subsequently on 2,4 , and $6 \mathrm{~h}$, and on d $1,2,7$, and 14 . Error bars indicate SEM. ${ }^{* *} P \leq 0.01,{ }^{*} P \leq 0.05$. 
venous administration of IL-8 (Hechtman et al., 1991; Van Zee et al., 1992). However, it was later demonstrated that the same granulocytes that were circulating when IL-8 was injected returned to the circulation (Ley et al., 1993). Measurements performed in that study showed that radiolabeled granulocytes transiently disappeared from the circulating pool, got trapped in the pulmonary microcirculation, and returned to the systemic circulation within 30 min after IL-8 challenge (Ley et al., 1993). Nevertheless, the increased percentage of circulating neutrophils measured in our study after intravenous injection of rbIL-8 suggests that there is a mixed population of granulocytes after IL-8 treatment, such that not only demargination but also release from the bone marrow reserve accounts for the neutrophilia seen after intravenous administration of rbIL-8. The results from the current study show that our purification method was effective and resulted in biologically active rbIL- 8 that can be used safely to modulate immune responses in cattle.

\section{CONCLUSIONS}

In the present study, we standardized the process of cloning, production, and purification of rbIL- 8 from bacterial culture. Subsequent column chromatography resulted in final purified yields of $1.2 \mathrm{mg}$ of $>95 \%$ pure rbIL-8 per liter of E. coli culture. Experiments were conducted to evaluate the safety of treating animals with rbIL- 8 and to assess its biological activity in vivo. No adverse effects on health and welfare were observed during the preliminary studies conducted in heifers, and lactating cows that were allocated to receive $1,125 \mu \mathrm{g}$ of rbIL-8 intravenously or via intrauterine administration. Intrauterine administration with $1,125 \mu \mathrm{g}$ of rbIL8 induced a 20 -fold increase in the proportion of PMN cells in uterine cytology, which was not observed in resin-purified lysate of $E$. coli BL21 that was not transfected with the plasmid coding for rbIL-8. Additionally, intravenous treatment with rbIL-8 has marked effects on white blood cell populations including general leukopenia between 2 and $4 \mathrm{~h}$ after intravenous injection and a rebound increase in neutrophil counts at $6 \mathrm{~h}$. These results show that this purification method is effective and results in biologically active rbIL- 8 that can be used safely to modulate immune responses in cattle.

\section{ACKNOWLEDGMENTS}

This work was partially supported by Zoetis Animal Health (Kalamazoo, MI) and by the Agriculture and Food Research Initiative Competitive Grant No. 201767015-26541 from the United States Department of
Agriculture National Institute of Food and Agriculture (Washington, DC).

\section{REFERENCES}

Baggiolini, M., A. Walz, and S. L. Kunkel. 1989. Neutrophil-activating peptide-1/interleukin 8, a novel cytokine that activates neutrophils. J. Clin. Invest. 84:1045-1049.

Brito, L. A., and M. Singh. 2011. COMMENTARY: Acceptable levels of endotoxin in vaccine formulations during preclinical research. J. Pharm. Sci. 100:34-37.

Caswell, J. L., D. M. Middleton, and J. R. Gordon. 1999. Production and functional characterization of recombinant bovine interleukin- 8 as a specific neutrophil activator and chemoattractant. Vet. Immunol. Immunopathol. 67:327-340.

Elliott, C. L., D. M. Slater, W. Dennes, L. Poston, and P. R. Bennett 2000. Interleukin 8 expression in human myometrium: Changes in relation to labor onset and with gestational age. Am. J. Reprod. Immunol. 43:272-277.

Gilbert, R. O., S. T. Shin, C. L. Guard, H. N. Erb, and M. Frajblat. 2005. Prevalence of endometritis and its effects on reproductive performance of dairy cows. Theriogenology 64:1879-1888.

Gleerup, K. B., P. H. Andersen, L. Munksgaard, and B. Forkman. 2015. Pain evaluation in dairy cattle. Appl. Anim. Behav. Sci. $171: 25-32$.

Goff, J. P., and R. L. Horst. 1997. Physiological changes at parturition and their relationship to metabolic disorders. J. Dairy Sci 80:1260-1268.

Gunnink, J. W. 1984a. Post-partum leucocytic activity and its relationship to caesarian section and retained placenta. Vet. Q. 6:55-57.

Gunnink, J. W. 1984b. Retained placenta and leucocytic activity. Vet. Q. 6:49-51.

Hammon, D. S., I. M. Evjen, T. R. Dhiman, J. P. Goff, and J. L. Walters. 2006. Neutrophil function and energy status in Holstein cows with uterine health disorders. Vet. Immunol. Immunopathol. $113: 21-29$.

Hechtman, D. H., M. I. Cybulsky, H. J. Fuchs, J. B. Baker, and M. A. Gimbore. 1991. Intravascular interleukin-8: An inhibitor of polymorphnuclear leukocyte accumulation at sites of acute inflammation. J. Immunol. 147:883-892.

Helle, M., J. P. Brakenhoff, E. R. De Groot, and L. A. Aarden. 1988. Interleukin 6 is involved in interleukin 1-induced activities. Eur. J. Immunol. 18:957-959.

Heuwieser, W., and E. Grunert. 1987. Steroid hormone profile in the afterbirth expulsion period of cattle. Dtsch. Tierarztl. Wochenschr. 94:311-314.

Hou, D., Z. Yan, J. Shi, W. Han, and Y. Zhang. 2005. Expression and one-step ion-exchange purification of (AAR)IL-8 (human IL-8 receptor antagonist). Protein Expr. Purif. 44:104-109.

Jagels, M. A., and T. E. Hugli. 1992. Neutrophil chemotactic factors promote leukocytosis. A common mechanism for cellular recruitment from bone marrow. J. Immunol. 148:1119-1128.

Johnson, J. L., H. Hong, J. Monfregola, and S. D. Catz. 2011. Increased survival and reduced neutrophil infiltration of the liver in Rab27a- but Not Munc13-4-deficient mice in lipopolysaccharideinduced systemic inflammation. Infect. Immun. 79:3607-3618. https://doi.org/10.1128/IAI.05043-11.

Kasimanickam, R., T. F. Duffield, R. A. Foster, C. J. Gartley, K. E. Leslie, J. S. Walton, and W. H. Johnson. 2004. Endometrial cytology and ultrasonography for the detection of subclinical endometritis in postpartum dairy cows. Theriogenology 62:9-23.

Kehrli, M. E., Jr., B. J. Nonnecke, and J. A. Roth. 1989. Alterations in bovine neutrophil function during the periparturient period. Am. J. Vet. Res. 50:207-214.

Kettelhut, I. C., and A. L. Goldberg. 1988. Tumor necrosis factor can induce fever in rats without activating protein breakdown in muscle or lipolysis in adipose tissue. J. Clin. Invest. 81:1384-1389. 
Kimura, K., J. P. Goff, M. E. Kehrli Jr., and T. A. Reinhardt. 2002. Decreased neutrophil function as a cause of retained placenta in dairy cattle. J. Dairy Sci. 85:544-550.

Laham, N., S. P. Brennecke, and G. E. Rice. 1999. Interleukin-8 release from human gestational tissue explants: Effects of gestation, labor, and chorioamnionitis. Biol. Reprod. 61:823-827.

Ley, K., J. B. Baker, M. I. Cybulsky, M. A. Gimbrone Jr., and F. W. Luscinskas. 1993. Intravenous interleukin- 8 inhibits granulocyte emigration from rabbit mesenteric venules without altering L-selectin expression or leukocyte rolling. J. Immunol. 151:6347-6357.

Li, B., and M. Sha, 2015. Successful High-Density Escherichia coli Fermentation Using the Eppendorf BioFlo ${ }^{\circledR} 320$ Advanced Bioprocess Control System. BioProcessing J. 20-24.

Manley, H. R., M. C. Keightley, and G. J. Lieschke. 2018. The neutrophil nucleus: An important influence on neutrophil migration and function. Front. Immunol. 9:2867. https://doi.org/10.3389/fimmu .2018.02867.

Mitchell, G. B., B. N. Albright, and J. L. Caswell. 2003. Effect of interleukin-8 and granulocyte colony-stimulating factor on priming and activation of bovine neutrophils. Infect. Immun. 71:1643-1649.

Morsey, M. A., Y. Popowych, J. Kowalski, G. Gerlach, D. Godson, M. Campos, and L. A. Babiuk. 1996. Molecular cloning and expression of bovine interleukin-8. Microb. Pathog. 20:203-212.

Mosmann, T. 1983. Rapid colorimetric assay for cellular growth and survival: Application to proliferation and cytotoxicity assays. J. Immunol. Methods 65:55-63.

Paape, M., J. Mehrzad, X. Zhao, J. Detilleux, and C. Burvenich. 2002. Defense of the bovine mammary gland by polymorphonuclear neutrophil leukocytes. J. Mammary Gland Biol. Neoplasia 7:109-121.

Persson Waller, K. 1997. Modulation of endotoxin-induced inflammation in the bovine teat using antagonists/inhibitors to leukotrienes, platelet activating factor and interleukin $1 \mathrm{~b}$. Vet. Immunol. Immunopathol. 57:239-251.

Peters, M. D., I. D. Silveira, and V. Fischer. 2015. Impact of subclinical and clinical mastitis on sensitivity to pain of dairy cows. Animal 9:2024-2028.

Roth, J. A., and M. L. Kaeberle. 1981. Isolation of neutrophils and eosinophils from the peripheral blood of cattle and comparison of their functional activities. J. Immunol. Methods 45:153-164.

Rothwell, N. J., A. J. Hardwick, and I. Lindley. 1990. Central actions of interleukin- 8 in the rat are independent of prostaglandins. Horm. Metab. Res. 22:595-596.
Sheldon, I. M., G. S. Lewis, S. LeBlanc, and R. O. Gilbert. 2006. Defining postpartum uterine disease in cattle. Theriogenology 65:1516-1530

Shi, Y., B. S. Kornovski, R. Savani, and E. A. Turley. 1993. A rapid, multiwell colorimetric assay for chemotaxis. J. Immunol. Methods 164:149-154.

Sigrell, J. A., P. Eklund, M. Galin, L. Hedkvist, P. Liljedahl, C. M. Johansson, T. Pless, and K. Torstenson. 2003. Automated multidimensional purification of tagged proteins. J. Struct. Funct. Genomics 4:109-114.

Stojkov, J., M. A. von Keyserlingk, J. N. Marchant-Forde, and D. M. Weary. 2015. Assessment of visceral pain associated with metritis in dairy cows. J. Dairy Sci. 98:5352-5361.

Tsukamoto, Y., S. Fukutani, Y. Takayama, H. Fukushima, H. Sagawa, and M. Mori. 1990. Characterization of leukocyte chemotactic activity of bacteriocin from Streptococcus mutans Rm-10. Inflammation 14:561-569.

Van Zee, K. J., E. Fischer, A. S. Hawes, C. A. Hebert, T. G. Terrell, J. B. Baker, S. F. Lowry, and L. L. Moldawer. 1992. Effects of intravenous IL-8 administration in nonhuman primates. J. Immunol. 148:1746-1752.

Watanabe, A., J. Hirota, S. Shimizu, S. Inumaru, and K. Kimura 2012. Single intramammary infusion of recombinant bovine interleukin-8 at dry-off induces the prolonged secretion of leukocyte elastase, inflammatory lactoferrin-derived peptides, and interleukin-8 in dairy cows. Vet. Med. Int. 2012:172072.

Zampronio, A. R., M. E. Hoadley, G. Luheshi, N. J. Rothwell, G. E. de Souza, and S. J. Hopkins. 2000. Interleukin (IL)-6 release and fever induced by a pre-formed pyrogenic factor (PFPF) derived from LPS-stimulated macrophages. Eur. Cytokine Netw. 11:589-596.

Zampronio, A. R., C. A. Silva, F. Q. Cunha, S. H. Ferreira, I. R. Pela, and G. E. Souza. 1995. Indomethacin blocks the febrile response induced by interleukin-8 in rabbits. Am. J. Physiol. 269:R1469R1474.

Zampronio, A. R., G. E. Souza, C. A. Silva, F. Q. Cunha, and S. H. Ferreira. 1994. Interleukin-8 induces fever by a prostaglandinindependent mechanism. Am. J. Physiol. 266:R1670-R1674.

Zhu, J., and S. G. Emerson. 2002. Hematopoietic cytokines, transcription factors and lineage commitment. Oncogene 21:3295-3313. 